\title{
1 A Bayesian model of metapopulation viability, with application to an
}

2 endangered amphibian

3

7 Geoffrey W. Heard

8 School of Botany, University of Melbourne, Victoria, AUSTRALIA 3010.

$9 \quad$ Ph: +61390357522

10 Email: heardg@unimelb.edu.au analysis'

\section{Michael A. McCarthy}

School of Botany, University of Melbourne, Victoria, Australia 3010.

Michael P. Scroggie P.O. Box 137, Heidelberg, Victoria, AUSTRALIA 3084

John B. Baumgartner

School of Botany, University of Melbourne, Victoria, Australia 3010.

Kirsten M. Parris

School of Botany, University of Melbourne, Victoria, Australia 3010.

Running title: A Bayesian SPOM

Article type: Biodiversity Research and Reviews

Keywords: amphibian, Bayesian, metapopulation, risk, regression, SPOM, viability.

This paper is a contribution to the special issue on: 'Perspectives and tools for conservation risk

Arthur Rylah Institute for Environmental Research, Department of Sustainability and Environment, 
Aim: Population viability analysis (PVA) is used to quantify the risks faced by species under alternative management regimes. Bayesian PVAs allow uncertainty in the parameters of the underlying population model to be easily propagated through to the predictions. We developed a Bayesian stochastic patch occupancy model (SPOM), and used this model to assess the viability of a metapopulation of the growling grass frog (Litoria raniformis) under different urbanisation scenarios.

34 Location: Melbourne, Victoria, Australia. colonisation. data to reduce parametric uncertainty. These advantages, and the ready availability of software to run 
54 Even the simplest conservation decisions may entail considerable uncertainty. The complexity of ecological processes and their inherently stochastic nature ensures that the response of populations or communities to particular management actions cannot be assessed in a purely mechanistic framework (Williams et al., 2002). Instead, conservation biologists turn to probabilistic approaches to explore the risks associated with particular management decisions. They seek to estimate the probability that an adverse event will occur in the future under a particular management scenario, given a mathematical model of the system, and known or plausible sources of stochasticity (Burgman et al., 1993). In many cases, the uncertainty captured by this approach relates only to the stochasticity of the modelled dynamics, but incorporating uncertainty in the model itself is also important. Doing so allows known uncertainty around the estimated risk to be considered in management decisions, and highlights areas where better knowledge of the system is required to reduce that uncertainty (Burgman, 2005).

Population viability analysis (PVA) remains an important form of risk analysis for the management of threatened species. PVA is the quantitative evaluation of factors that influence population growth and persistence for single species (Boyce, 1992; Lindenmayer et al., 1995; McCarthy \& Possingham, in press). Early incarnations were focussed on single populations, and sought to estimate the probability of extinction for small populations subject to environmental, demographic and genetic stochasticity (Soulé, 1987; Beissinger, 2002). Contemporary PVAs are designed for more complicated problems, and incorporate more complicated dynamics. Because many threatened species display some population sub-division, and because habitat fragmentation is near ubiquitous as a threatening process, PVAs that incorporate multiple interacting populations (metapopulations) have now largely replaced PVAs focussed on a single population (Morris \& Doak, 2002). These models allow migration between the component populations, and may also incorporate such things as 
spatially-variable demographic parameters, habitat dynamics, and spatially-correlated disturbance regimes (McCarthy, 2009). The application of these models is now less concerned with the outright risk of extinction, and instead focuses on comparative risks. Given a set of options for future management, analysts seek to quantify and compare the relative risk of extinction under each (e.g., Lindenmayer \& Possingham, 1996; Akçakaya \& Raphael, 1998; Drechsler et al., 2003).

Alternatively, they may seek to optimise viability subject to budget constraints. In these situations, the candidate set of management options is not predefined; rather the analyst is charged with identifying the combination of management actions that minimizes the probability of extinction for a set budget (e.g., Moilanen \& Cabeza, 2002).

While the use of PVA to predict the relative risk of extinction should be more robust to uncertainty than predictions of outright extinction risk (McCarthy et al., 2003), propagating uncertainty through to the model predictions remains desirable for such analyses (Morris \& Doak, 2002). Numerous uncertainties may influence a given PVA, but parametric uncertainty is ubiquitous. Parametric uncertainty arises from sampling variation, observer bias, and sampling error in empirically derived population models, and through differences of opinion for models parameterised using expert knowledge (McGowan et al., 2011). Despite its ubiquity, accounting for parametric uncertainty in PVAs has proven technically challenging, and somewhat contentious (Wade, 2002). Bayesian approaches are becoming an increasingly popular means of performing PVAs as a result, because they allow straightforward propagation of parametric uncertainty through to model predictions (Ludwig, 1996; Wade, 2002; McCarthy, 2007). Bayesian statistical methods represent uncertainty in model parameters by using probability distributions, with the width of a parameter's distribution representing the imprecision of the associated estimate (McCarthy, 2007). Importantly, Bayesian methods recognise that information about a parameter accumulates over time. The prior distribution represents what is known about a parameter before considering the latest set of data. The information 
103 in the data about the parameters is combined with the prior using Bayes' rule, generating the 104 posterior distribution (McCarthy, 2007). Propagating parametric uncertainty in Bayesian PVA is simply a matter of simulating the dynamics of the focal population/s for combinations of parameter values drawn from their joint posterior distributions, thereby providing a distribution for the chosen viability metric that represents uncertainty due to imprecision in the parameters, as well as that due to stochasticity of the modelled dynamics.

In the metapopulation literature, Bayesian approaches to PVA have primarily been pursued in the context of stochastic patch occupancy models, or SPOMs. These models are designed for the situation in which population extinction is commonplace, and persistence of the metapopulation relies upon a balance between the rate of population extinction and the rate of colonisation of vacant habitat patches (Sjögren-Gulve \& Hanski, 2000; Hanski, 2002). Rather than attempting to model the internal demographic processes of the constituent populations, SPOMs describe the stochastic changes in population occurrence arising from population extinction and colonisation (Sjögren-

117 Gulve \& Hanski, 2000; Hanski, 2002). The most well known and widely applied SPOM is the 118 incidence function model (IFM) pioneered by Hanski (1994). Hanski’s model incorporates the key effects of patch area and isolation on the probabilities of extinction and colonisation (see Hanski, 120 1999), and was the first to allow simulation of the dynamics of real metapopulations subject to habitat loss, degradation and fragmentation. Bayesian formulations of the IFM have been offered by O’Hara et al. (2002), ter Braak \& Etienne (2003), Harrison et al. (2011) and Risk et al. (2011).

Nevertheless, the application of the IFM to conservation planning has been criticized. The reasons are various, ranging from arguments against the generality of the patch area and isolation paradigm (Pellet et al., 2007), to technical concerns such as the need to assume stationarity of extinction and colonisation rates when estimating the parameters of the model using occupancy data (Thomas, 
128 1994). While these concerns are by no means fatal to the IFM, they do point to a need for alternative 129 approaches when the shortcomings of the IFM are not trivial. One option is to model the 130 probabilities of population extinction and colonisation directly (Sjögren-Gulve \& Ray, 1996;

131 Sjögren-Gulve \& Hanski, 2000; Morris \& Doak, 2002). The idea is simple - given an adequate 132 sample of extinction and colonisation events, one may build regression models of relationships 133 between the probabilities of extinction and colonisation and particular patch- and landscape-level 134 variables thought to influence these processes. Arbitrarily complex linear and non-linear 135 relationships can be incorporated, providing considerable flexibility for model development. As 136 demonstrated by Sjögren-Gulve \& Ray (1996), the resulting regression models for the probabilities 137 of population extinction and colonisation can be used to simulate the dynamics of real 138 metapopulations in the same way as the IFM, and therefore offer the same opportunities to predict 139 the viability of real metapopulations subject to habitat change (see also Sjögren-Gulve \& Hanski, 140 2000; Morris \& Doak, 2002).

Here we present a Bayesian version of the regression approach introduced by Sjögren-Gulve \& Ray 143 (1996), and use it to assess the viability of a metapopulation of an endangered frog from southern 144 Australia. This is, to our knowledge, the first time a Bayesian formulation of Sjögren-Gulve \& Ray’s approach to metapopulation viability analysis has been presented. We demonstrate how the Bayesian formulation allows parametric uncertainty to be easily propagated through to predictions of 147 metapopulation viability under differing scenarios of habitat loss and creation.

149 Methods 
151 Before proceeding to the technical aspects of our approach, we first briefly expand on the theoretical basis of SPOMs. Classical metapopulation theory assumes networks of spatially discrete populations connected by infrequent dispersal. Within these networks, populations are prone to extinction given sensitivity to demographic, genetic and environmental stochasticity, but these extinctions may be offset by colonisation of vacant habitat patches. Population extinction and colonisation are thus random events, but the probabilities of extinction and colonisation vary among patches and over time. The probability of extinction is modeled as a function of population size and, in some cases, immigration rate (the 'rescue effect' sensu Brown \& Kodric-Brown, 1977). The probability of colonisation is modeled as a function of the number of immigrants. Spatially-realistic SPOMs are based on the premise that particular patch- and landscape-scale variables may be used as surrogates for population size and immigration rate (Hanski, 1994; Sjögren-Gulve \& Ray, 1996). The most basic of these relationships are encapsulated by the IFM: population size scales with patch area, and immigration rate depends on the number, proximity and size of neighboring populations ('isolation', or, conversely, 'connectivity'). However, additional variables are likely to be important determinants of population size and immigration rate in some circumstances. When patch quality is highly variable, even small patches may have high carrying capacities (Fleishman et al., 2002). Similarly, the surrounding landscape could be more or less permeable to migrants, in which case the number of immigrants arriving at a patch will only partly reflect its proximity to neighbouring populations (Wiens, 1997).

As above, the approach of Sjögren-Gulve \& Ray (1996) is predicated on the use of regression models to describe relationships between the probabilities of extinction and colonisation and patchand landscape-scale variables thought to influence population size and immigration rate. Applying the logit link, the probability of extinction ( $(\varepsilon)$ for population $i$ at time $t$ is modelled as:

$$
\operatorname{logit}\left(\varepsilon_{i, t}\right)=\alpha_{\varepsilon}+\beta_{\varepsilon 1}\left(X_{1, i, t-1}\right)+\beta_{\varepsilon 2}\left(X_{2, i, t-1}\right)+\ldots+\beta_{\varepsilon N}\left(X_{N, i, t-1}\right),
$$


where $\alpha_{\varepsilon}$ is the intercept term, $X_{1, i, t-1}, X_{2, i, t-1}$, and $X_{N, i, t-1}$ are values for the relevant patch- and

landscape-scale variables at the preceding time-step, and $\beta_{\varepsilon 1,} \beta_{\varepsilon 2}$, and $\beta_{\varepsilon N}$ are regression coefficients.

Likewise, the probability of colonisation $(\gamma)$ for patch $i$ at time $t$ is modelled as:

$$
\operatorname{logit}\left(\gamma_{i, t}\right)=\alpha_{\gamma}+\beta_{\gamma 1}\left(Z_{1, i, t-1}\right)+\beta_{\gamma 2}\left(Z_{2, i, t-1}\right)+\ldots+\beta_{\gamma N}\left(Z_{N, i, t-1}\right)
$$

Sjögren-Gulve \& Ray (1996) describe how the parameters of Eq. 1 and Eq. 2 may be estimated using maximum likelihood when data on occupancy turnover between two time-steps are available. However, as Sjögren-Gulve \& Ray acknowledge, their approach assumes that the focal species is detected without error; an assumption that may be problematic for many species (Tyre et al., 2003; MacKenzie et al., 2006). Recent advances in occupancy modelling circumvent this problem. Mackenzie et al. (2003) provided an approach in which maximum likelihood estimates of the probabilities of extinction and colonisation may be derived from multi-year occupancy datasets, whilst accounting for imperfect detection. Although the data requirements are slightly different (repeat survey data within years being required to jointly estimate the probability of detection), the approach of Mackenzie et al. (2003) is similar to that of Sjögren-Gulve \& Ray (1996), because the probabilities of extinction and colonisation may be modelled as functions of covariates using the logit link function.

Royle \& Kéry (2007) provided a Bayesian state-space formulation of the dynamic occupancy model of Mackenzie et al. (2003) which allows the posterior distribution of the parameters of equations 1 and 2 to be derived using Markov chain Monte Carlo (MCMC) sampling. Royle \& Kéry (2007) implemented their method using WinBUGS (Lunn et al., 2000), but it is also directly transferable to OpenBUGS (the open-source version of WinBUGS; Thomas et al., 2006) and may also be implemented in JAGS (an alternative program for fitting Bayesian models using MCMC sampling; Plummer, 2003) with only minor alteration. 
202 Once estimates of the parameters of equations 1 and 2 have been derived using MCMC sampling,

203 they can be inserted into the standard equation for changes in patch occupancy to define a SPOM. The standard equation is (MacKenzie et al., 2003):

$$
O_{i, t+1}=o_{i, t}\left(1-\varepsilon_{i, t}\right)+\left(1-o_{i, t}\right) \gamma_{i, t}
$$

where $O_{i, t+1}$ is the probability of occupancy of patch $i$ at time $t+1, o_{i, t}$ is the occupancy status of patch $i$ at time $t$ ( 1 if occupied, 0 otherwise), and $\varepsilon_{i, t}$ and $\gamma_{i, t}$ are the probabilities of extinction and colonisation for patch $i$ at time $t$, as defined by equations 1 and 2 . Thus, the probability of occupancy at time $t+1$ is equal to the probability of colonisation $\left(\gamma_{i, t}\right)$ if the patch was unoccupied at the previous time step, and is equal to the probability of persistence $\left(1-\varepsilon_{i, t}\right)$ if the patch was occupied. Using the resulting model to simulate the dynamics of the metapopulation over a chosen time period is straightforward. For one time step to the next, the process is as follows: (i) estimate the probability of occupancy for each patch at the second time-step $\left(O_{i, t+1}\right)$, according to the occupancy status in the first time step $\left(o_{i, t}\right)$ and the probabilities of population extinction $\left(\varepsilon_{i, t}\right)$ and colonisation $\left(\gamma_{i, t}\right)$; (ii) set occupancy of each patch at the second time-step as the outcome of a Bernoulli trial with probability $O_{i, t+1}$, and; (iii) record the occupancy status of each patch at the second time-step. In order to incorporate parametric uncertainty, these simulations are simply repeated for each of the sample of parameter values derived from the MCMC algorithm.

\section{By running many simulations for each parameter combination and recording the number of occupied} patches through time for each simulation, one can estimate of the probability of extinction or quasiextinction for the metapopulation through time (Hanski, 2002). For each parameter combination, the probability of extinction is simply the proportion of simulations in which the metapopulation reached zero occupied patches. Similarly, the probability of quasi-extinction is estimated as the proportion of simulations in which the metapopulation declined below some threshold patch occupancy (e.g., $<5$ 
occupied patches). Of course, alternate measures of metapopulation viability could also be derived

227 from the simulations, such as the expected minimum number of occupied patches. This measure is

228 equivalent to the expected minimum population size in demographic PVAs, and gives a better

229 indication of the propensity for decline when the risk of extinction is small (McCarthy \& Thompson,

230 2001). Regardless of the metric used, uncertainty may be presented as the full distribution of

231 estimates across the parameter combinations, or through a summary of this distribution, such as the

232 mean and $95 \%$ credible interval.

234 Case study

235 The growling grass frog (Litoria raniformis) is an endangered frog that occurs throughout south236 eastern Australia (Pyke, 2002). In the vicinity of Melbourne, the capital city of Victoria, urban 237 expansion is a key threat to this species. Numerous remnant populations occur in areas slated for 238 urban growth, and associated wetland loss, degradation and fragmentation may place 239 metapopulations of L. raniformis at considerable risk of extinction (Heard et al., 2012a, b).

240 Developing a model of the extinction and colonisation dynamics of L. raniformis has subsequently 241 been identified as a priority for understanding the species' conservation requirements (Heard et al., 242 2012b).

244 We built a Bayesian regression-based SPOM for L. raniformis using occupancy data from 167 245 wetlands monitored across northern Melbourne between the 2001/2002 and 2006/2007 breeding 246 seasons (Heard et al., 2012b). Wetlands included slow-flowing pools along streams, swamps, ponds, 247 farm dams and quarry pits. All wetlands were surveyed on between one and four occasions in the 248 first and last years of the study (with the exception of four wetlands that were destroyed before the 249 final year), and a subset surveyed on up to 10 occasions in the intervening years. A total of 1380 250 surveys were completed. 
252 We began by identifying patch- and landscape-scale variables that influence the extinction and 253 colonisation dynamics of L. raniformis. For the purposes of this paper, we focussed on a model in 254 which the probability of extinction was a function of effective wetland area, aquatic vegetation cover 255 and connectivity, and the probability of colonisation was a function of connectivity alone. Effective 256 area $\left(A_{e f f}\right)$ was defined as:

$$
A_{\text {eff } i}=\frac{\log \left(A_{i}\right) \times H_{i}}{H_{\text {max }}},
$$

258 where $A_{i}$ is the surface area of wetland $i, H_{i}$ is the hydroperiod of wetland $i$ measured on an ordinal scale between 1 (temporarily fills only during high rainfall periods) and 4 (permanently inundated, regardless of rainfall variability), and $H_{\max }$ is the maximum hydroperiod score (4). Adjusting wetland area for hydroperiod accounted for the fact that wetland carrying capacity should be closely tied to fluctuations in water-level for $L$. raniformis, due to its highly aquatic nature (Heard et al., 2008; Heard et al., 2012b). Aquatic vegetation cover is considered a key microhabitat for both larval and adult $L$. raniformis, and therefore represents a key patch quality variable for the species (Hamer $\&$ Organ, 2008; Heard et al., 2008; Wassens et al., 2010). Aquatic vegetation cover for each wetland $\left(V_{i}\right)$ was defined as the mean cover of emergent, submergent and floating aquatic vegetation. After

$$
S_{i, t}=\sum w_{i, j} \times o_{j, t}
$$
where $w_{i, j}$ is a weighting function, and $o_{j, t}$ is the occupancy status of each neighbour $j$ in year $t$ (one if the wetland is occupied, zero if not). For years $2-6$, calculation of connectivity was complicated by the fact that some wetlands were not surveyed in those years, whilst others had been destroyed.

272 When occupancy data were not available for a wetland in a given year, the outcome of the nearest preceding survey for that wetland was used instead. Wetlands that had been destroyed were simply removed from connectivity calculations for the years following their destruction. The weighting 
275 function, $w_{i, j}$, defines a negative power relationship between the probability of dispersal from 276 wetland $i$ to $j$ and the Euclidean distance between their centres $\left(d_{i, j}\right.$, at $10 \mathrm{~m}$ increments). This 277 function, derived by Heard et al. (2012b) using mark-recapture data, was:

$$
w_{i, j}=0.10026 d_{i, j}^{-0.719877} \text {. }
$$

279 Connectivity $\left(S_{i, t}\right)$ was log-transformed for further analyses.

281 Using the approach of Royle \& Kéry (2007), we fitted a dynamic occupancy model to these data in which the logits of the probabilities of extinction and colonisation were modelled as additive, linear functions of the above variables. The logit of the probability of detection was set as an additive, linear function of survey effort (person minutes), date (days since October 1) and time of day (day or night) following Heard et al. (2012b). Vague, normally-distributed priors (N[0, 0.001]) were used for all model parameters. Convergence was assessed by visually inspecting the histories of two Markov chains with over-dispersed initial values. Convergence was achieved within 25,000 MCMC samples.

We extracted the subsequent 5000 estimates of the parameters of the model. Model fitting was completed with $O p e n B U G S$ v. 3.0.3, called from $R$ v. 2.12 .0 (R Development Core Team, 2012) using the add-in package BRugs v. 0.5-3 (Ligges, 2008). Code for running the analysis is provided in Appendix S1 of the Supporting Information. Table S1 gives summary statistics for the posterior distributions of the model parameters, and Fig. S1 shows the fitted relationships.

The parameter estimates were used to assess the viability of a metapopulation of L. raniformis located in Donnybrook, on Melbourne's northern fringe. This metapopulation occupies a reasonably discrete cluster of 30 wetlands, including pools along the Merri and Kalkallo Creeks, and farm dams within $500 \mathrm{~m}$ of these creeks (Fig. 1). Many of these wetlands were included in the broader study of 
and $V$ for wetlands that were not surveyed by Heard et al. (2012b) were acquired in a subsequent study (Heard \& McCarthy, 2012). The only other information required to simulate the dynamics of the metapopulation were the coordinates of wetland centres and the initial occupancy status of each wetland. For simplicity, we assumed all wetlands were occupied at the initial time-step.

The dynamics of the Donnybrook metapopulation were simulated for a period of 30 years under three broad scenarios. The first (referred to hereafter as 'current conditions') entailed no change in wetland number or condition over this period (Fig. 1). In the second scenario, we deleted dams in accordance with urban growth plans for Donnybrook (DPCD, 2009). These plans involve the maintenance of riparian reserves for conservation purposes and for public parkland, although the size of these reserves is yet to be finalised. We trialled riparian reserves ranging from $500 \mathrm{~m}$ either side of both creeks, to $100 \mathrm{~m}$ either side, at $100 \mathrm{~m}$ increments (Fig. 1). In the final scenario, we assessed the ability of replacement wetlands to offset the losses expected under a $200 \mathrm{~m}$ riparian reserve scenario.

One to four pairs of wetlands were placed in the riparian reserve, next to central, high-quality patches (Fig. 1). Each new wetland was set to have the same features: $A=1250$ sq. $\mathrm{m}, H=3, V=60 \%$. These schemes sought to create clusters of populations which would display high probabilities of occupancy through time. We assumed that the dynamics of L. raniformis within created wetlands could be adequately described by our model, as the majority of lentic wetlands monitored in the previous occupancy study were artificial (farm dams, ponds and quarries).

For each of these scenarios, we ran 500 simulations for each of the 5000 combinations of the model parameter estimates. For each simulation run for each combination of parameter estimates, we recorded the minimum number of occupied wetlands across the 30 year time period. The mean minimum number of occupied wetlands (minocc) was calculated by averaging over the 500 simulations for each scenario and parameter estimate combination. We defined the metapopulation 
as having gone quasi-extinct during a simulation run if fewer than three wetlands were occupied at any time-step. The probability of quasi-extinction $(q e)$ for each scenario and parameter estimate combination was then simply the proportion of the 500 simulations in which quasi-extinction occurred. Change in the probability of quasi-extinction $(\Delta q e)$ and mean minimum number of occupied wetlands ( $\Delta$ minocc $)$ was calculated for each scenario and parameter estimate as:

$$
\Delta q e_{i}=q e_{i}-q e_{c},
$$

and

$$
\Delta \text { minocc }_{i}=\text { minocc }_{i}-\text { minocc }_{c}
$$

where $q e_{i}$ is the probability of quasi-extinction for scenario $i, q e_{c}$ is the probability of quasiextinction assuming no change from current conditions, minocc $c_{i}$ is the mean minimum number of occupied wetlands for scenario $i$, and minocc $_{c}$ is the mean minimum number of occupied wetlands assuming no change from current conditions. Simulations were run in $R$, using code provided in Appendix S2.

\section{Results}

There was considerable uncertainty in the estimates of the minimum number of occupied wetlands (minocc) for the Donnybrook metapopulation of $L$. raniformis given either the maintenance of current conditions or the maintenance of a $500 \mathrm{~m}$ riparian reserve (Fig. 2). The $95 \% \mathrm{CI}$ for minocc was $\sim 3-18$ for both scenarios. Uncertainty in this metric declined with increasing levels of urbanisation due to the reduced size of the metapopulation. Importantly, both the mean estimate and upper $95 \%$ CI of minocc fell substantially between the $500 \mathrm{~m}$ to $400 \mathrm{~m}$ reserve scenarios (Fig. 2); a result of the fact that the $400 \mathrm{~m}$ reserve entails the loss of three large farm dams (see Fig. 1). There was also little difference between the $300 \mathrm{~m}$ to $100 \mathrm{~m}$ reserve scenarios for minocc (Fig. 2), as most dams lost across these scenarios were small, shallow and lacked aquatic vegetation. Mean estimates 
suggest that the probability of quasi-extinction (qe, defined as less than three occupied wetlands) would be $<0.2$ for this metapopulation over 30 years under all scenarios (Fig. 2). However, the uncertainty in these estimates was very large for the $400 \mathrm{~m}$ to $100 \mathrm{~m}$ reserve scenarios; the $95 \% \mathrm{CI}$ being at least $0-0.7$ in each case. As with minocc, qe increased between the $500 \mathrm{~m}$ and $400 \mathrm{~m}$ reserves, and stabilised thereafter.

354 Change in both the minimum number of occupied wetlands ( $\Delta$ minocc) and the probability of quasiextinction $(\Delta q e)$ was negligible for the $500 \mathrm{~m}$ riparian reserve scenario (mean estimates $=-0.674$ and 0.003 respectively; Fig. 3). The rate of change in $\Delta$ minocc was greatest between the $500 \mathrm{~m}$ to $400 \mathrm{~m}$ reserve scenarios, reflecting the stabilisation of minocc for the narrower reserves (Fig. 3). However, uncertainty in $\Delta$ minocc displayed the opposite trend to minocc, with wider 95\% CIs for the narrower reserves. While estimates of $\Delta$ minocc show that the metapopulation will decline with increasing habitat loss, the $95 \% \mathrm{CI}$ for $\Delta q e$ overlapped zero for all reserve scenarios (Fig. 3). Hence, in each case, there was a small chance that the probability of quasi-extinction would not change from current conditions. Uncertainty in $\Delta q e$ increased with decreasing reserve sizes, in accord with $\Delta$ minocc (Fig. $3)$. under this scenario. Only with the creation of eight wetlands was the lower bound of the $95 \%$ CI for $\Delta$ minocc above zero (0.36). Estimates of $\Delta q e$ present a slightly different picture of the response of this metapopulation to wetland creation (Fig. 4). In all cases, the mean estimates of $\Delta q e$ were close to 
373 zero (-0.018-0.024), suggesting little change in metapopulation viability from current conditions.

374 However, there was a rapid transition in the distribution of estimates of $\Delta q e$ from the first scenario 375 (two new wetlands) to the third (six new wetlands). Under the first scenario, an increase in the probability of quasi-extinction was predicted for almost all parameter combinations $(95 \% \mathrm{CI}$ of $\Delta q e$

$377=-0.002-0.264)$. The reverse was true when six new wetlands were added $(95 \% \mathrm{CI}$ of $\Delta q e=-0.230$ 378 0). This rapid transition in $\Delta q e$ stemmed from the strategic placement of the new wetlands, and the 379 low threshold for quasi-extinction. By locating the new wetlands in pairs close to high-quality 380 patches, clusters of persistent populations were created. With three such clusters (six new wetlands), 381 there was a sharp drop in the chance of falling below the quasi-extinction threshold of three extant 382 populations.

\section{Discussion}

385 Bayesian models allow straightforward propagation of parametric uncertainty through to predictions derived from these models. Previous studies have shown how Bayesian PVA models may be used to derive probability distributions for the chance of extinction or the expected minimum (meta)population size (Ludwig, 1996; Wade, 2002; ter Braak \& Etienne, 2003; McCarthy, 2007).

Here we have shown that uncertainty in the relative increase or decrease in these measures under alternate management scenarios is also easily calculated using Bayesian models. This is important for decision-making because the magnitude of uncertainty can influence the perceived impacts of management, and the relative performance of different options. For example, in our case study, the mean estimates of the minimum number of wetlands occupied by L. raniformis ( $\Delta$ minocc) and the probability of quasi-extinction $(\Delta q e)$ did not change markedly between the $300 \mathrm{~m}, 200 \mathrm{~m}$ and $100 \mathrm{~m}$ riparian reserve scenarios (because the wetlands lost under these scenarios were small and of relatively poor quality). As these scenarios would differ markedly in their cost to implement, it may 
be concluded that benefits conferred by the larger reserves are not economically justified. However, our analysis also demonstrates that there is considerable and increasing uncertainty about the change in both $\Delta$ minocc and $\Delta q e$ as the reserve size narrows, with large decreases in metapopulation viability being plausible (Fig. 3). Documenting this uncertainty allows managers to account for it in their decisions. A risk-tolerant manager might be prepared to choose narrower reserves based on the central tendency, whereas a risk-averse manager striving for negligible impacts might impose a large riparian reserve (e.g., 500 m; Fig. 3) or demand considerable offsetting works (e.g., six to eight new wetlands; Fig. 4).

Of course, it is preferable to subject these sorts of decision-making processes to analyses in which the objectives are explicit, and uncertainty is directly integrated. Although we have not illustrated it to set some standard for the trajectory of the metapopulation, and identify the strategy that minimizes the chance of breaching that standard (a form of satisficing; Simon, 1982; Burgman, 2005; McCarthy et al., 2010). The task for the analyst is then to calculate the chance of breaching the threshold for the 
option. In a Bayesian analysis, these probabilities may be calculated as the proportion of the posterior distribution of the viability metric that breaches the chosen threshold.

While the above analyses are useful for identifying the best strategy when there is a single decision to be made, management of metapopulations can involve recurrent decisions about how to manage the constituent populations. Stochastic dynamic programming (SDP) is ideally suited for these situations (Possingham, 1996; Westphal et al., 2003). Nevertheless, current examples of SDP do not include uncertainty in the parameters of the underlying metapopulation model. Including parametric uncertainty produces an optimization problem in which the parameter estimates, the management actions and the response of the metapopulation to those actions are all variable. While including each of these sources of uncertainty in SDP would be a valuable advance, it may prove technically challenging, or even impossible, due to the size and complexity of the optimization problem.

As well as propagating parametric uncertainty through to predictions and the resulting decisionmaking process, Bayesian approaches to PVA are also useful for identifying parameters that represent critical uncertainties of the underlying model. Critical uncertainties are not just highly uncertain components of the system being modelled. They are uncertainties which, if resolved, can change management decisions (Rumpff et al., 2011; Runge, 2011). One option for identifying parameters that represent critical uncertainties in a Bayesian PVA is to perform analyses akin to traditional sensitivity analyses. Simulations could be run for multiple combinations of parameters drawn from their joint posterior distributions, and the management scenario which is optimal for each draw identified from the simulations. The change in the optimal scenario across the posterior distribution of each parameter could then be quantified, and used as measure of the influence of uncertainty in each parameter on management decisions. When only two management scenarios are considered, the influence of parameter uncertainty on the optimal scenario could assessed using 
446 logistic regression, with the response variable being which management strategy is optimal 447 (McCarthy et al., 1995).

449 Bayesian methods also provide a clear path to increasing the precision of parameters that represent 450 critical uncertainties. Because they incorporate existing knowledge by specifying a prior distribution, 451 Bayesian approaches explicitly recognise that information about a parameter accumulates over time 452 (McCarthy, 2007). In the context of PVA, one can envision building an initial model using vague 453 priors and available data (as we have done here), and then periodically updating the model as 454 monitoring data accumulate, using the posterior from the previous model as the prior in the new one. 455 When both management and monitoring are directed at reducing uncertainty about key parameters, this should help resolve critical uncertainties. For example, in our case study, it was apparent that the relationship between connectivity and the probability of colonisation for L. raniformis was highly uncertain at the upper levels of connectivity, because wetlands with high connectivity were rarely unoccupied (see Fig. S1). Should an interrogation of the model reveal that this uncertainty influences the optimal management strategy, one could ensure that at least some created wetlands are placed very close to currently occupied wetlands. Subsequent monitoring of metapopulations manipulated in this way will deliver data about the rate of colonisation of highly connected wetlands, which, when combined with existing information, will reduce uncertainty about this relationship, and reduce management indecision.

This study focused on a regression-based stochastic patch occupancy model (SPOM) following Sjögren-Gulve \& Ray (1996). The regression-based approach has advantages over alternatives when data are available to model extinction and colonisation probabilities directly (Sjögren-Gulve \& Hanski, 2000; Morris \& Doak, 2002). Model structure is highly flexible, meaning that the inclusion 470 of patch quality variables (in particular) is easily accomplished. There is also no need to assume 
stationarity of extinction and colonisation rates to parameterize the model using occupancy data. As well as providing a Bayesian implementation, we have shown that the approach of Sjögren-Gulve \& Ray (1996) may also be extended to account for imperfect detection, given recent advances in occupancy modeling (Mackenzie et al. 2003, 2006; Royle \& Kéry 2007). This is important, because failing to account for false absences may bias estimates of the parameters of SPOMs, and undermine their predictive power (Moilanen, 2002). Nevertheless, we did not fully account for imperfect detection in our case study. For the purposes of calculating connectivity $\left(S_{i, t}\right)$, we assumed that the occupancy status of each wetland by L. raniformis was determined perfectly in years for which survey data were available, and that occupancy status had not changed from previous years when survey data were unavailable. These assumptions were tolerable in this study, with cumulative probabilities of detection averaging $>0.8$ when survey data were available, and missing data being largely restricted to sites whose occupancy status was unlikely to have changed through time. Nevertheless, allowing for imperfect detection and changes in occupancy status when calculating $S_{i, t}$ will be appealing in many situations. Both can be accommodated in Bayesian occupancy models (Royle \& Dorazio, 2008; Bled et al., 2011; Risk et al., 2011), because the occupancy status of each patch at each time-step is simulated for each iteration of the MCMC algorithm, enabling stochastic realizations of $S_{i, t}$ to be calculated (see Appendix S1). However, this comes at considerable computational cost when the number of patches is large. The decision to include stochastic realizations of $S_{i, t}$ in the model therefore requires consideration of the likely scale of bias induced by its exclusion, and the computational costs of including it.

Bayesian approaches to PVA explicitly incorporate and propagate uncertainty in models of population processes, allowing informed decision-making and identification of critical uncertainties in the underlying model. For these reasons, and because of the ready availability of software to fit Bayesian models, we expect interest in Bayesian approaches to PVA to continue to rise. When 
496 focused on the comparative risks associated with alternate management options, and particularly 497 when coupled with formal decision analyses that identify the optimal approach given a particular 498 objective, these analyses have the potential to provide valuable direction to the management of 499 threatened species.

501 Acknowledgements

502 Funding for this project was provided by the Victorian Department of Sustainability and 503 Environment (DSE), the Australian Research Council Centre of Excellence for Environmental 504 Decisions, and an Australian Research Council Linkage Grant (LP0990161) with the Australian 505 Research Centre for Urban Ecology, DSE, Growling Grass Frog Trust Fund, Melbourne Museum, 506 Melbourne Water and Parks Victoria. MM was supported by an Australian Research Council Future 507 Fellowship. We thank Adam Muir and Kim Lowe (DSE) for their support of the project. Stefano 508 Canessa (University of Melbourne) provided coding advice and assistance. Charles Todd (DSE) and 509 two anonymous reviewers offered useful critiques of an earlier version.

References

512 Akçakaya, H.R. \& Raphael, M.G. (1998) Assessing human impact despite uncertainty: viability of the northern spotted owl metapopulation in the northwestern USA. Biodiversity and Conservation, 7, 875-894.

Beissinger, S.R. (2002) Population viability analysis: past, present and future. Population Viability Analysis (ed. by S.A. Beissinger and D.R. McCullough), pp. 5-17. The University of Chicago Press, Chicago. 
Bled, F., Royle, J.A. \& Cam, E. (2011) Hierarchical modeling of an invasive spread: the Eurasian Collared-Dove Streptopelia decaocto in the United States. Ecological Applications, 21, 290302.

Boyce, M.S. (1992) Population Viability Analysis. Annual Review of Ecology and Systematics, 23, 481-506.

Brown, J.H. \& Kodric-Brown, A. (1977) Turnover rates in insular biogeography: effect of immigration on extinction. Ecology, 58, 445-449.

Burgman, M.A. (2005) Risks and Decisions for Conservation and Environmental Management. Cambridge University Press, Cambridge.

Burgman, M.A., Ferson, S. \& Akçakaya, H.R. (1993) Risk Assessment in Conservation Biology. Chapman and Hall, London.

DPCD (2009) Delivering Melbourne's Newest Sustainable Communities. Victorian Department of Planning and Community Development, Melbourne.

Drechsler, M. \& Burgman, M.A. (2004) Combining population viability analysis with decision analysis. Biodiversity and Conservation, 13, 115-139.

Drechsler, M., Frank, K., Hanski, I., O'Hara, R.B. \& Wissel, C. (2003) Ranking metapopulation extinction risk: from patterns in data to conservation management decisions. Ecological Applications, 13, 990-998.

Fleishman, E., Ray, C., Sjögren-Gulve, P., Boggs, C.L. \& Murphy, D.D. (2002) Assessing the roles of patch quality, area, and isolation in predicting metapopulation dynamics. Conservation Biology, 16, 706-716.

Hamer, A.J. \& Organ, A. (2008) Aspects of the ecology and conservation of the growling grass frog Litoria raniformis in an urban-fringe environment, southern Victoria. Australian Zoologist, 34, 393-407. 
Hanski, I. (1994) A practical model of metapopulation dynamics. Journal of Animal Ecology, 63, $151-162$.

Hanski, I. (1999) Metapopulation Ecology. Oxford University Press, Oxford.

Hanski, I. (2002) Metapopulations of animals in highly fragmented landscapes and population viability analysis. Population Viability Analysis (ed. by S.R. Beissinger and D.R. McCullough), pp. 86-108. The University of Chicago Press, Chicago.

Harrison, P.J., Hanski, I. \& Ovaskainen, O. (2011) Bayesian state-space modeling of metapopulation dynamics in the Glanville fritillary butterfly. Ecological Monographs, 81, 581-598.

Heard, G.W. \& McCarthy, M.A. (2012) Metapopulation viability of the Growling Grass Frog in Melbourne's urban growth areas. Report to the Victorian Department of Sustainability and Environment. University of Melbourne, Melbourne.

Heard, G.W., Robertson, P. \& Scroggie, M.P. (2008) Microhabitat preferences of the endangered Growling Grass Frog (Litoria raniformis) in southern Victoria, Australia. Australian Zoologist, 34, 414-425.

Heard, G.W., Scroggie, M.P. \& Malone, B.S. (2012a) The life history and decline of the threatened Australian frog, Litoria raniformis. Austral Ecology, 37, 276-284.

Heard, G.W., Scroggie, M.P. \& Malone, B.S. (2012b) Classical metapopulation theory as a useful paradigm for the conservation of an endangered amphibian. Biological Conservation, 148, 156-166.

Ligges, U. (2008) BRugs. R Foundation for Statistical Computing. http://cran.rproject.org/web/packages/BRugs/

Lindenmayer, D.B. \& Possingham, H.P. (1996) Ranking conservation and timber management options for Leadbeater's Possum in southeastern Australia using population viability analysis. Conservation Biology, 10, 235-251. 
Lindenmayer, D.B., Burgman, M.A., Akçakaya, H.R., Lacy, R.C. \& Possingham, H.P. (1995) A review of the generic computer programs ALEX, RAMAS/space and VORTEX for modelling the viability of wildlife metapopulations. Ecological Modelling, 82, 161-174.

Ludwig, D. (1996) Uncertainty and the assessment of extinction probabilities. Ecological Applications, 6, 1067-1076.

Lunn, D.J., Thomas, A., Best, N. \& Spiegelhalter, D. (2000) WinBUGS - a Bayesian modelling framework: concepts, structure, and extensibility. Statistics and Computing, 10, 325-337.

MacKenzie, D.I., Nichols, J.D., Hines, J.E., Knutson, M.G. \& Franklin, A.B. (2003) Estimating site occupancy, colonisation, and local extinction when a species is detected imperfectly. Ecology, 84, 2200-2207.

MacKenzie, D.I., Nichols, J.D., Royle, J.A., Pollock, K.H., Bailey, L.L. \& Hines, J.E. (2006) Occupancy Estimation and Modeling: Inferring Patterns and Dynamics of Species Occurrence. Elsevier, San Diego.

McCarthy, M.A. (2007) Bayesian Methods for Ecology. Cambridge University Press, New York.

McCarthy, M.A. (2009) Spatial population viability analysis. Spatial Conservation Prioritization: Quantitative Methods and Computational Tools (ed. by A. Moilanen, K.A. Wilson and H.P. Possingham), pp. 122-134. Oxford University Press, New York.

McCarthy, M.A. \& Thompson, C. (2001) Expected minimum population size as a measure of threat. Animal Conservation, 4, 351-355.

McCarthy, M.A. \& Possingham, H.P. (in press) Population viability analysis. Encyclopedia of Environmetrics, 2nd edition (ed. by A.H. El-Shaarawi and W.W. Piegorsch). WileyBlackwell.

McCarthy, M.A., Burgman, M.A. \& Ferson, S. (1995) Sensitivity analysis for models of population viability. Biological Conservation, 73, 93-100. 
McCarthy, M.A., Andelman, S.J. \& Possingham, H.P. (2003) Reliability of relative predictions in population viability analysis. Conservation Biology, 17, 982-989.

McCarthy, M.A., Thompson, C.J., Hauser, C., Burgman, M.A., Possingham, H.P., Moir, M.L., Tiensin, T. \& Gilbert, M. (2010) Resource allocation for efficient environmental management. Ecology Letters, 13, 1280-1289.

McGowan, C.P., Runge, M.C. \& Larson, M.A. (2011) Incorporating parametric uncertainty into population viability analysis models. Biological Conservation, 144, 1400-1408.

Moilanen, A. (2002) Implications of empirical data quality to metapopulation model parameter estimation and application. Oikos, 96, 516-530.

Moilanen, A. \& Cabeza, M. (2002) Single-species dynamic site selection. Ecological Applications, 12, 913-926.

Morris, W.F. \& Doak, D.F. (2002) Quantitative Conservation Biology: Theory and Practice of Population Viability Analysis. Sinauer Associates, Sunderland.

O'Hara, R.B., Arias, E., Toivonen, H. \& Hanski, I. (2002) Bayesian analysis of metapopulation data. Ecology, 83, 2408-2415.

Pellet, J., Fleishman, E., Dobkin, D.S., Gander, A. \& Murphy, D.D. (2007) An empirical evaluation of the area and isolation paradigm of metapopulation dynamics. Biological Conservation, 136, $483-465$.

Plummer, M. (2003) JAGS: a program for analysis of Bayesian graphical models using Gibbs sampling. 3rd International Workshop on Distributed Statistical Computing (DSC 2003), pp. 1-10. Vienna, Austria.

Possingham, H.P. (1996) Decision theory and biodiversity management: how to manage a metapopulation. Frontiers of Population Ecology (ed. by R.B. Floyd, A.W. Sheppard and P.J. De Barro), pp. 391-398. CSIRO Publishing, Melbourne. 
614 Possingham, H.P., Lindenmayer, D.B. \& Tuck, G.N. (2002) Decision theory for population viability

615

616 analysis. Population Viability Analysis (ed. by S.R. Beissinger and D.R. McCullough), pp. 470-489. University of Chicago Press, Chicago.

Pyke, G.H. (2002) A review of the biology of the southern bell frog Litoria raniformis (Anura: Hylidae). Australian Zoologist, 32, 32-48.

R Development Core Team (2012) R: A language and environment for statistical computing. $\mathrm{R}$ Foundation for Statistical Computing. http://www.r-project.org/

Risk, B.B., de Valpine, P. \& Beissinger, S.R. (2011) A robust-design formulation of the incidence function model of metapopulation dynamics applied to two species of rails. Ecology, 92, 462474.

Royle, J.A. \& Kéry, M. (2007) A Bayesian state-space formulation of dynamic occupancy models. Ecology, 88, 1813-1823.

Royle, J.A. \& Dorazio, R.M. (2008) Hierarchical Modeling and Inference in Ecology: The Analysis of Data from Populations, Metapopulations and Communities. Academic Press, San Diego.

Rumpff, L., Duncan, D.H., Vesk, P.A., Keith, D.A. \& Wintle, B.A. (2011) State-and-transition modelling for adaptive management of native woodlands. Biological Conservation, 144, 1224-1236.

Runge, M.C. (2011) An introduction to adaptive management for threatened and endangered species. Journal of Fish and Wildlife Management, 2, 220-233.

Simon, H.A. (1982) Models of Bounded Rationality. MIT Press, Cambridge.

Sjögren-Gulve, P. \& Ray, C. (1996) Using logistic regression to model metapopulation dynamics: large-scale forestry extirpates the pool frog. Metapopulations and Wildlife Conservation (ed. by D.R. McCullough), pp. 111-137. Island Press, Washington.

Sjögren-Gulve, P. \& Hanski, I. (2000) Metapopulation viability analysis using occupancy models. Ecological Bulletins, 48, 53-71. 
Soulé, M.E. (ed.) (1987) Viable Populations for Conservation. Cambridge University Press, New York.

ter Braak, C.J.F. \& Etienne, R.S. (2003) Improved Bayesian analysis of metapopulation data with an application to a tree frog metapopulation. Ecology, 84, 231-241.

Thomas, A., O'Hara, B., Ligges, U. \& Sturtz, S. (2006) Making BUGS Open. $R$ News, 6, 12-17.

Thomas, C.D. (1994) Difficulties in deducing dynamics from static distributions. Trends in Ecology and Evolution, 9, 300.

Tyre, A.J., Tenhumenberg, B., Field, S.A., Neijalke, D., Parris, K. \& Possingham, H.P. (2003) Improving precision and reducing bias in biological surveys: estimating false-negative error rates. Ecological Applications, 13, 1790-1801.

Wade, P.R. (2002) Bayesian population viability analyses. Population Viability Analysis (ed. by S.A. Beissinger and D.R. McCullough), pp. 213-238. The University of Chicago Press, Chicago.

Wassens, S., Hall, A., Osborne, W. \& Watts, R.J. (2010) Habitat characteristics predict occupancy patterns of the endangered amphibian Litoria raniformis in flow-regulated flood plain wetlands. Austral Ecology, 35, 944-955.

Westphal, M.I., Pickett, M., Getz, W.M. \& Possingham, H.P. (2003) The use of stochastic dynamic programming in the optimal landscape reconstruction for metapopulations. Ecological Applications, 13, 543-555.

Wiens, J.A. (1997) Metapopulation dynamics and landscape ecology. Metapopulation Biology: Ecology, Genetics and Evolution (ed. by I. Hanski and M.E. Gilpin), pp. 43-62. Academic Press, San Diego.

Williams, B.K., Nichols, J.D. \& Conroy, M.J. (2002) The Analysis and Management of Animal Populations. Academic Press, San Diego. 


\section{Supporting Information}

664

665 Additional Supporting Information may be found in the online version of this article:

667 Appendix S1 \{Code for fitting the occupancy turnover model and extracting parameter estimates \}

668 Appendix S2 \{Code for simulating the dynamics of the Donnybrook metapopulation\}

669 Figure S1 \{Relationships between the probability of extinction and effective wetland area, aquatic

670 vegetation cover and connectivity, and between the probability of colonisation and connectivity\}

671 Table S1 \{Posteriors of the coefficients of the regression models for extinction and colonisation\}

672

673 As a service to our authors and readers, this journal provides supporting information supplied by the

674 authors. Such materials are peer-reviewed and may be re-organized for online delivery, but are not

675 copy-edited or typeset. Technical support issues arising from supporting information (other than

676 missing files) should be addressed to the authors.

677

678 
Fig. 1. The Donnybrook metapopulation of Litoria raniformis under alternate management scenarios. The top panel displays the distribution of wetlands (grey circles) under current conditions, where 'wetlands' include pools along the Merri and Kalkallo Creeks (represented by the black trace) and adjacent farm dams. As an example of different urbanisation scenarios considered, the middle panel displays the distribution of wetlands given urbanisation (shading) of all land beyond a $400 \mathrm{~m}$ reserve either side of each stream. The bottom panel displays the distribution of wetlands given a $200 \mathrm{~m}$ riparian reserve and the construction of eight new wetlands (black circles). Numbering of the new wetlands is the modelled sequence. Co-ordinates follow the Australian Map Grid system (with WGS 1994). Wetland sizes are scaled to their effective areas, but their shapes are stylised.

Fig. 2. The minimum number of occupied wetlands (minocc) and the probability of quasi-extinction (qe) for the Donnybrook metapopulation of Litoria raniformis over 30 years under five urbanisation scenarios. The scenarios represent maintenance of current conditions (CC) or five possible riparian reserve scenarios given urbanisation. These scenarios entail reservation of all land within $500 \mathrm{~m}$ of both streams in the area ('500 m'), reservation of all land within $400 \mathrm{~m}$ of both streams ('400 m'), and so on down to $100 \mathrm{~m}$ either side of both streams. Points represent the mean estimates, and vertical lines represent the $95 \%$ credible intervals.

Fig. 3. Change in both the minimum number of occupied wetlands $(\Delta m i n o c c)$ and the probability of quasi-extinction $(\Delta q e)$ for the Donnybrook metapopulation of Litoria raniformis over 30 years under five urbanisation scenarios. The scenarios represent possible widths of a riparian reserve system, as in Fig. 1. A score of zero on the y-axis represents no change in qe or minocc from current conditions. Points represent the mean estimates, and vertical lines represent the $95 \%$ credible intervals. 
703 Fig. 4. Change in the minimum number of occupied wetlands ( $\Delta$ minocc) and the probability of 704 quasi-extinction $(\Delta q e)$ for the Donnybrook metapopulation of Litoria raniformis over 30 years, given 705 reservation of all land within $200 \mathrm{~m}$ of both streams in this area, and the creation of either two, four, 706 six or eight new wetlands. A score of zero on the y-axis represents no change in qe or minocc from 707 current conditions. Points represent the mean estimates, and vertical lines represent the $95 \%$ credible 708 intervals. 

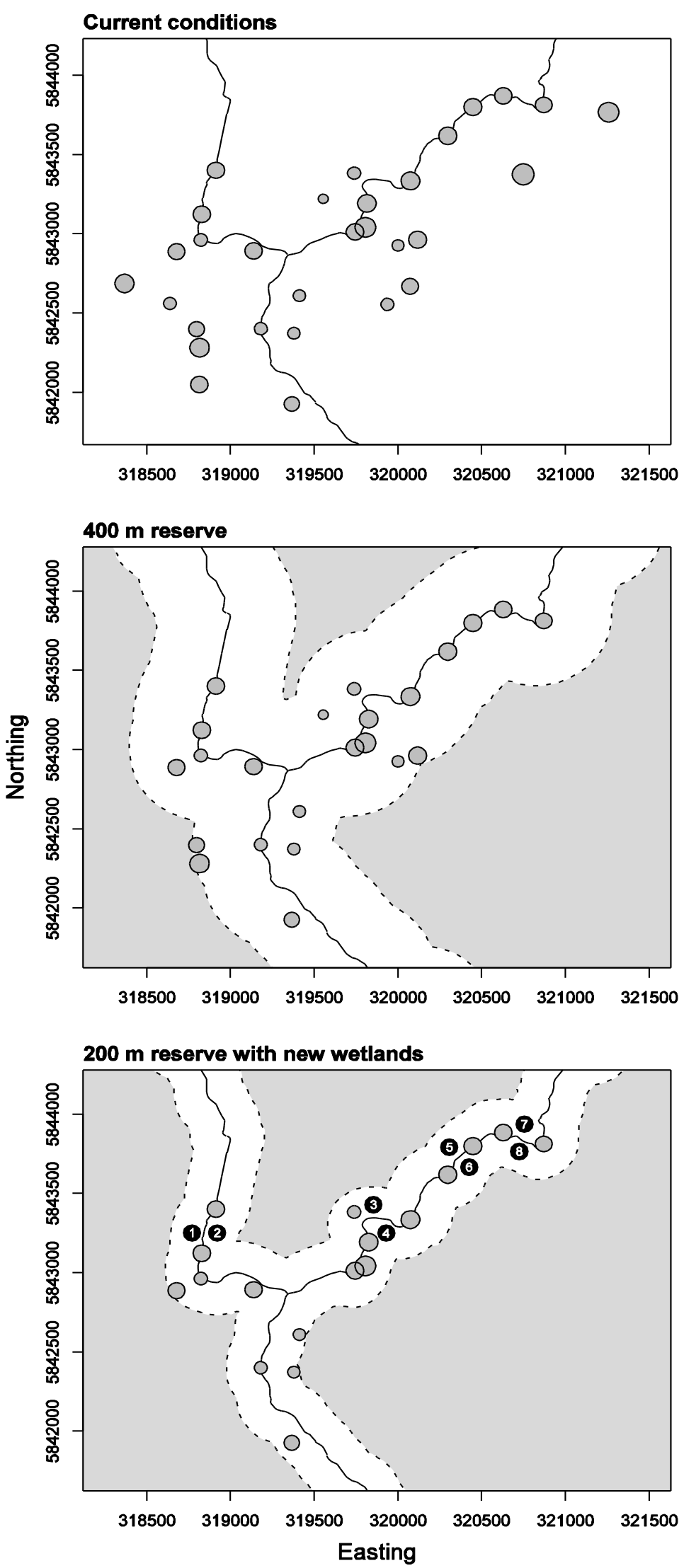

711 Fig. 1 

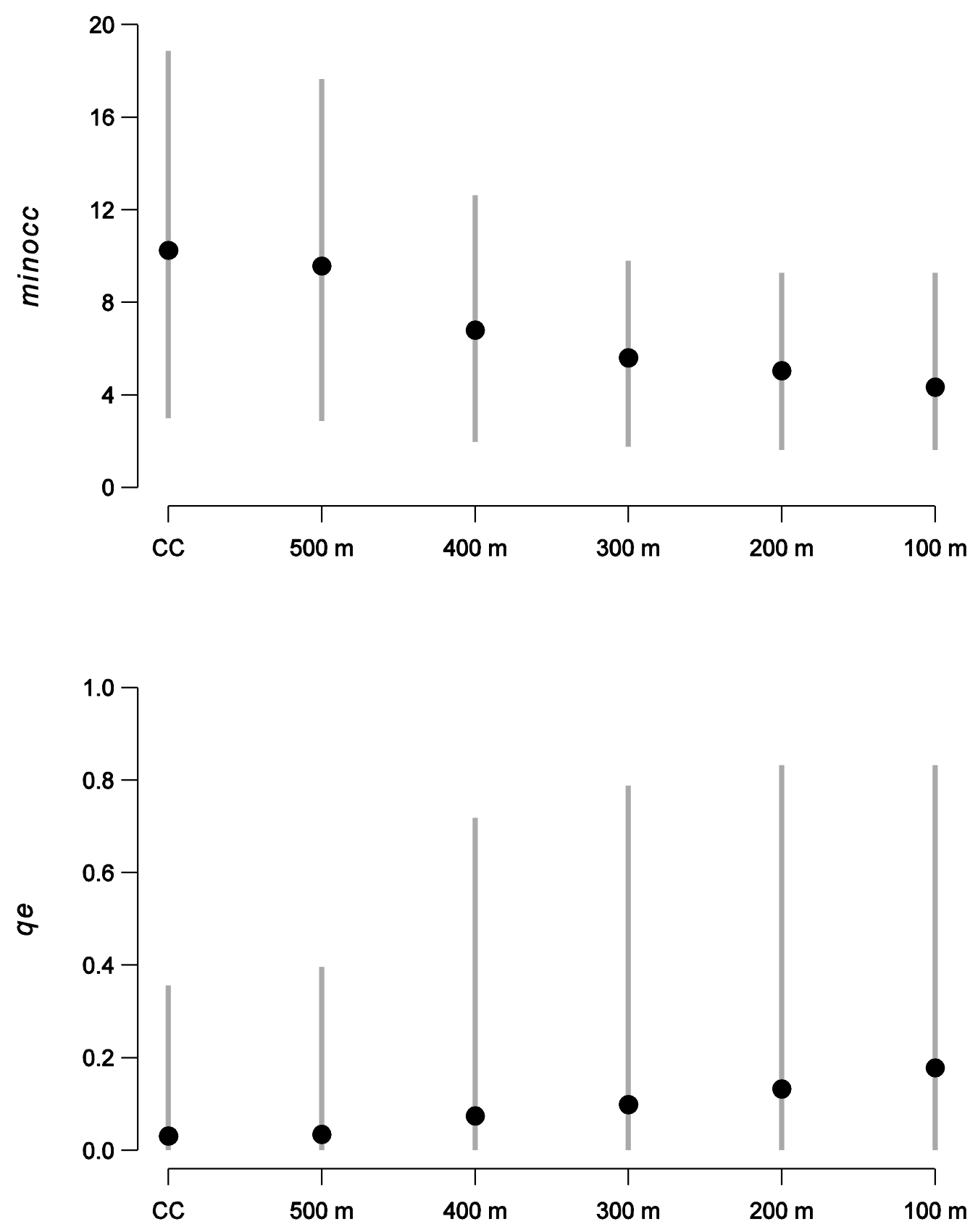

Fig. 2 

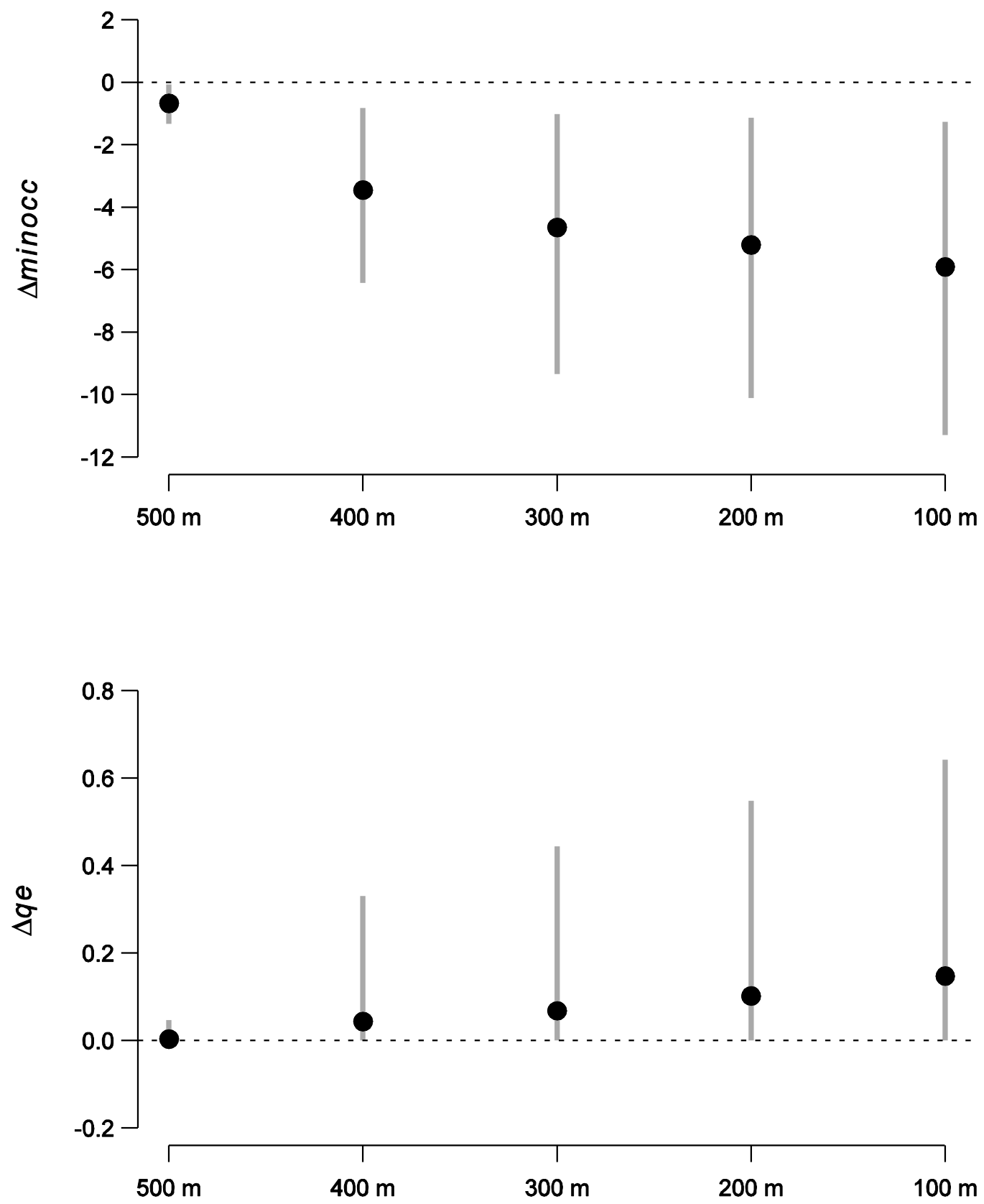

Scenario

720

721

722

723

$724 \quad$ Fig. 3 

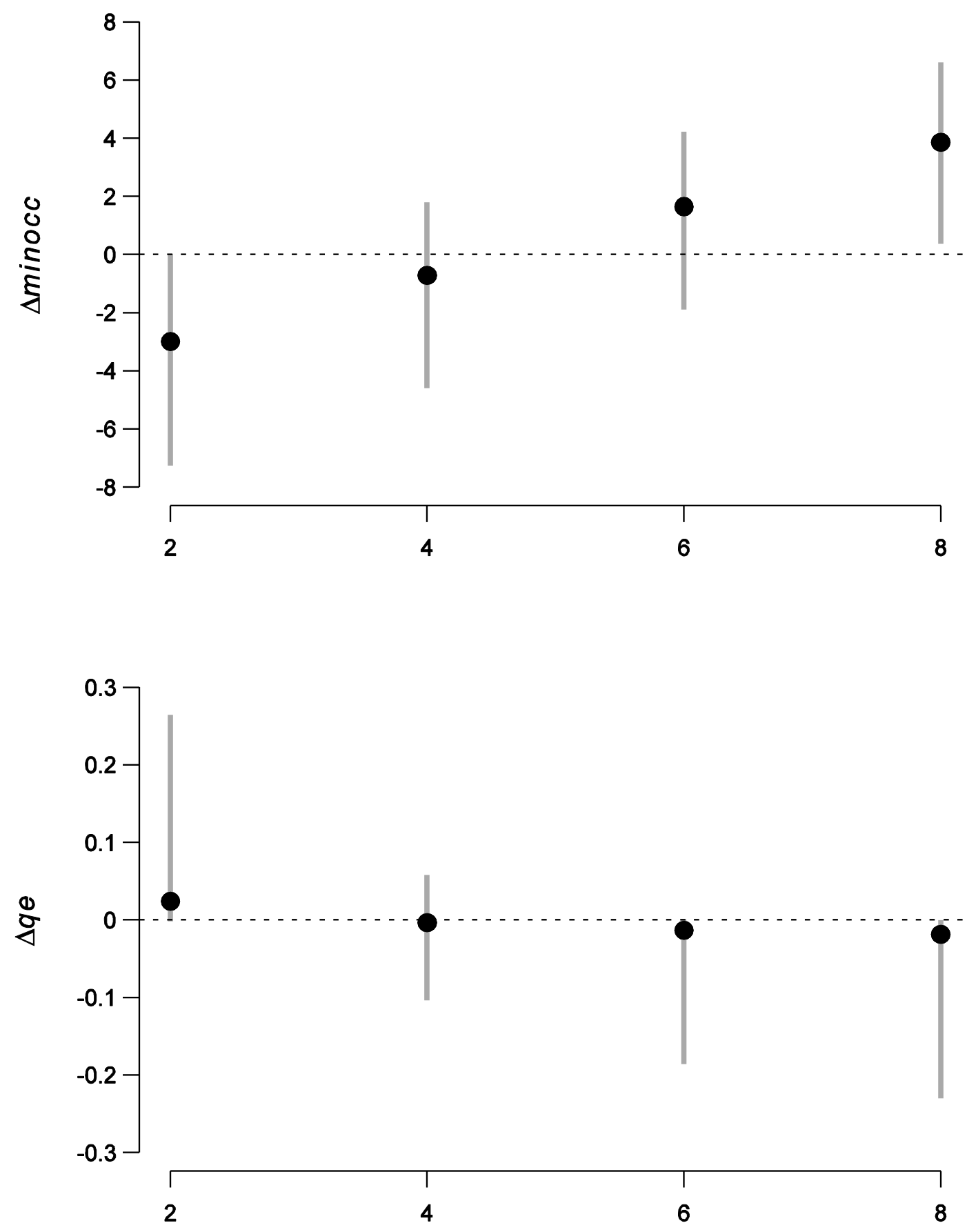

Number of new wetlands

$730 \quad$ Fig. 4 